\title{
Epidermolysis bullosa: a case report
}

This article was published in the following Dove Press journal: Chronic Wound Care Management and Research

\author{
Caroline E Fife' \\ Raphael A Yaakov ${ }^{2}$ \\ Thomas E Serena ${ }^{2}$ \\ 'CHI St. Luke's Health, Wound Care \\ Clinic, The Woodlands, TX, USA; \\ ${ }^{2}$ SerenaGroup, Clinical Research, \\ Cambridge, MA, USA
}

Correspondence: Thomas E Serena SerenaGroup, 125 Cambridge Park Drive, Suite 30 I Cambridge, MA 02 I40, USA Tel + I 6179455225

Email serena@serenagroups.com

\begin{abstract}
Epidermolysis bullosa (EB), often referred to as the butterfly disease, is a group of rare genetic conditions characterized by skin that is delicate and fragile as butterfly wings. The skin blisters in response to friction, minor injury, or trauma. In certain types of EB, other organs, such as the esophagus, can also be affected, and secondary complications may require multiple interventions. While there has been significant progress in classifying the disease identifying genes and proteins involved - there have been few advances in the treatment of the disease. The care of the EB patient focuses on management of symptoms, protecting the skin, and preventing complications. In this case report, the use of a multivalent wound-healing ointment (Terrasil ${ }^{\circledR}$ ) was evaluated in a 60 -year-old patient with a history of junctional EB. A polymerase chain reaction-based culturing was utilized to quantitatively test for bacteria and fungi at baseline and follow-up visits. Pain assessment and wound area were also documented at each visit. Following the application of the wound care ointment, there was a reduction in wound surface area on central (96\%) and distal mid-back (92\%) by treatment visit three, and there was a $96 \%$ reduction on the left shoulder blade ulcer by treatment visit four. Moreover, there was a noticeable drop in the percentage of bacteria detected by polymerase chain reaction. The wound care ointment was also effective in eliminating the fungal species and reducing pain, itching, blistering, and cracking around the wound.
\end{abstract}

Keywords: mechanobullous disorders, adjunctive therapy, bioburden, therapeutic management

\section{Introduction}

Epidermolysis bullosa (EB) is a group of rare genetic conditions characterized by fragile skin that blisters in response to friction, minor injury, or trauma. The National EB Registry estimates the incidence of EB at 19 cases per million live births and prevalence is approximated to be eight per 1 million within the USA. ${ }^{1}$ The manifestation of symptoms can range from mild to severe, based on the types/subtypes of EB and the inheritance pattern (autosomal dominant versus recessive); however, even patients with a relatively mild form may experience moderate to severe pain, discomfort from pruritus and complications, including infection, sepsis, dehydration, malnutrition, anemia, and the development of skin malignancies. The challenges associated with management and care of EB can pose a significant burden on the patient and caregiver.

While clinical presentation or the extent of ulceration may vary according to the distinctive phenotypes, skin blistering is a common characteristic for all types of EB. Provisional diagnosis can often be made by clinical observation; however, EB is most 
definitively diagnosed by histological analysis of skin biopsy. Immunofluorescence (IF), antigenic mapping, and transmission electron microscopy are key diagnostic tools used to confirm and identify a particular subtype of EB. ${ }^{2}$ Prenatal diagnosis can also be accomplished using amniocentesis or chorionic villus sampling as early as the first trimester of pregnancy. ${ }^{3}$

Treatment options for patients with EB are limited. The primary aim of the treatment is to protect the skin, reduce blistering, prevent complications, and promote healing. The prognosis of EB can vary dependent on the subtype of the disorder. While some patients have normal life expectancies, others can be at major risk of death during the first few years of life. ${ }^{4}$ For those who survive into adulthood, life can be severely limited.

Understanding of the mechanisms underlying the spectrum of clinical phenotypes of EB has greatly improved with advances in molecular and cell biology. A number of early studies broadly defined the major types of EB and linked the etiology to a misregulated or defective enzyme or a defect in a structural component of the skin or tissue. ${ }^{5,6}$ The association between the disorder and the cytoskeletal network was drawn with the application of electron microscopy. ${ }^{7}$ Moreover, the composition and structural details of the proteins that play a role in the pathogenesis of EB have been further refined in recent years: plakophilin 1, desmoplakin, keratin 5, keratin 14, plectin, $\alpha 6 \beta 4$-integrin, laminin 332, collagen XVII, and collagen VII are essential for the structural integrity of cytoskeleton of keratinocytes and basement membrane zone. ${ }^{8-11}$ Mutations in the genes that code for these structural proteins can cause EB.

\section{Case report}

A 60-year-old Caucasian male with junctional EB presented to Catholic Health Initiatives (CHI) St. Luke's Wound Care Clinic with a history of persistent non-healing ulcers. The ulcers were widespread, covering approximately one-third of the patient's body. The ulcerations on his shoulders, lower abdomen, back, thighs, and knees have been persistent since birth. The patient had a positive family history of EB. He had undergone resection of a large squamous cell carcinoma on the left lateral leg. This procedure was complicated by further tissue breakdown related to minor skin trauma during his recuperation.

Over the years, the patient has developed extensive experience caring for his wounds. He reported use of numerous dressing, including two of his favorites, PolyMem (Ferris Mfg. Corp, Fort Worth, TX, USA) and Xeroform (DeRoyal, Knoxville, TN, USA). He had tried a wide variety of ointments, ranging from over-the-counter products, such as
Vaseline (Unilever, Trumbull, CT, USA), topical steroids, Bacitracin, Neomycin, and Polymyxin B (Johnson \& Johnson, New Brunswick, NJ, USA), to prescription medication, including Mupirocin Calcium (GSK, Research Triangle Park, NC, USA) and Silver Sulfadiazine (Pfizer, New York, NY, USA). The patient also complained of unintentional weight loss and that his pain was not completely under control (8 on a Numeric Rating Scale of 0-10) despite the use of topical lidocaine (Roxane Laboratories, Columbus, OH, USA) and a number of oral analgesics, including hydrocodone/acetaminophen (Mallinckrodt, Dublin, Ireland) and methadone (Roxane Laboratories). He was also taking oral cephalexin (Advancis Pharmaceutical, Germantown, MD, USA).

On examination, it was observed that ulcerations were pink at the base with mild to moderate serosanguinous drainage; there was no foul odor. The periwound skin was erythematous. A polymerase chain reaction (PCR)-based culturing obtained revealed a variety of gram-positive bacteria, which typically colonize open skin, along with three different fungi including Volutella colletotrichoides, Phoma moricola, and Candida zeylanoides.

He received sample of Terrasil ${ }$, a novel multivalent wound care ointment (Aspiera Medical, Woonsocket, RI, USA). The ointment provides moisture management with jojoba seed oil and organic beeswax to achieve long-lasting moisturization. Xeroform (DeRoyal) and PolyMem (Ferris Mfg. Corp) were used as secondary dressings. In addition, it was recommended that the patient take ARGINAID ${ }^{\circledR}$ (Nestlé, Vevey, Switzerland), a nutritional supplement for individuals with wounds that include a blend of L-arginine and vitamins $C$ and $\mathrm{E}$. The ulcers on left shoulder blade, and central and distal mid-back were assessed and measured every 1-2 months. The goal of the treatment was to decrease the level of pain, increase granulation tissue formulation, and control bacterial burden.

The patient has a lifetime of experience in caring for body wide ulcers. He possesses exceptional knowledge of proper wound care. Additionally, he was instructed to wash and dry the affected area before the application of Terrasil ${ }^{\circledR}$ (Aspiera Medical). The ointment was applied daily with a Q-tip and the patient received a total of 332 applications. The patient reported a reduction in pain, itching, blistering, and cracking around the wound area. The follow-up PCR indicated mild bacterial load, and no fungal species were detected. The final PCR showed there was a drop in the percentage of Corynebacterium striatum, Corynebacterium simulans, Corynebacterium tuberculostearicum, Staphylococcus lugdunensis, and Staphylococcus epidermidis; however, the level of Staphylococcus aureus was particularly higher (Figure 1). 
There was a reduction in wound surface area on central and distal mid-back after the application of the wound care ointment. By treatment visit three, the ulcerations on central and distal mid-back had decreased by $96 \%$ and $92 \%$, respectively (Figures 2 and 3). At visit five, there was a 96\% reduction in wound area on the left shoulder blade (Figure 4). While a reduction in wound surface area was noted in response to the wound care ointment; not unexpectedly, some new ulcerations were formed. The formation of new wounds and the gradual increase of wound surface area were likely due to the progression of EB.

\section{Discussion}

Recent studies have shed light on a number of macromolecules which are essential for the cohesion of the skin and its resistance to shearing forces. It has furthered our

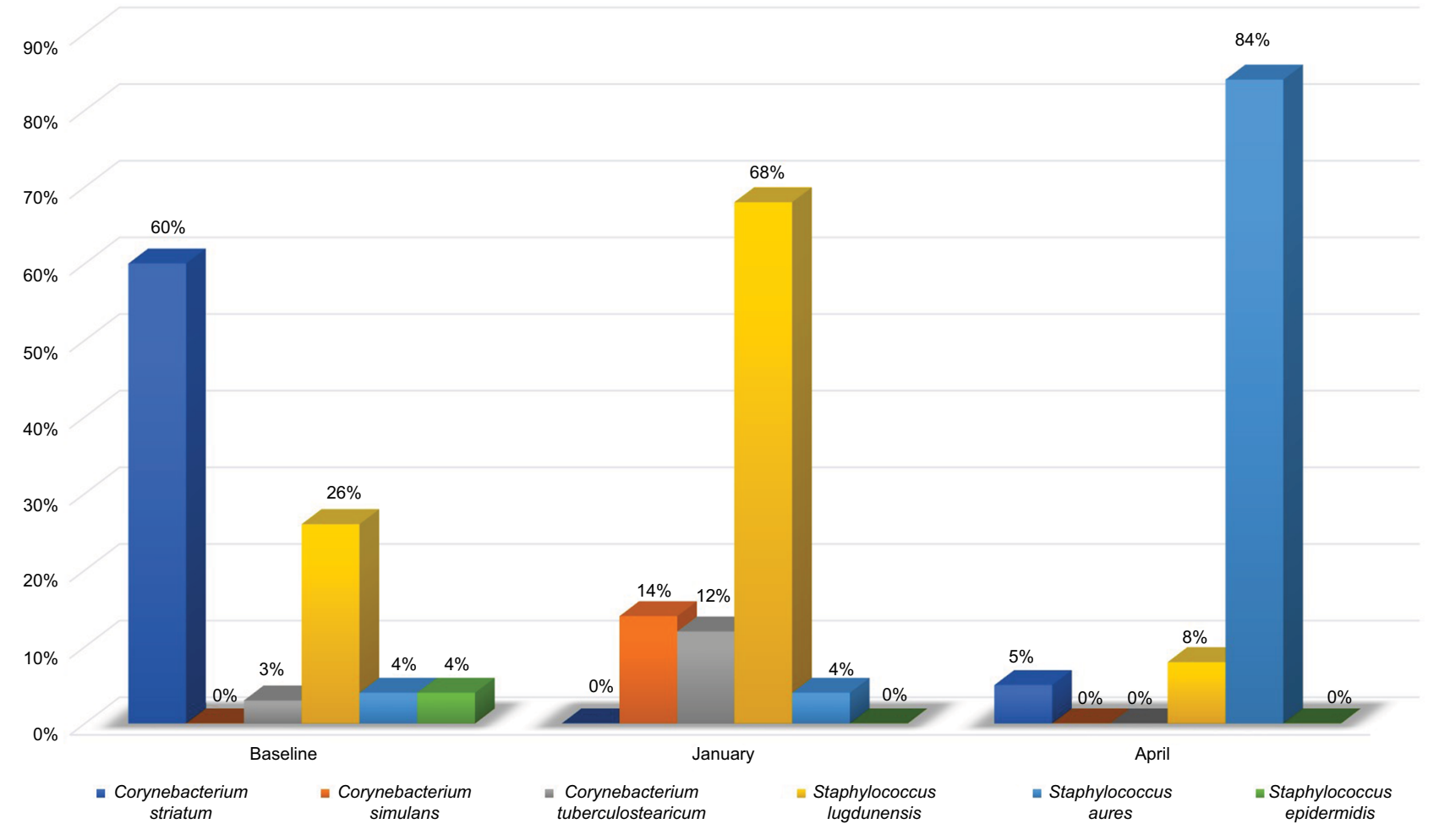

Figure I Estimated percentage of bacteria present.
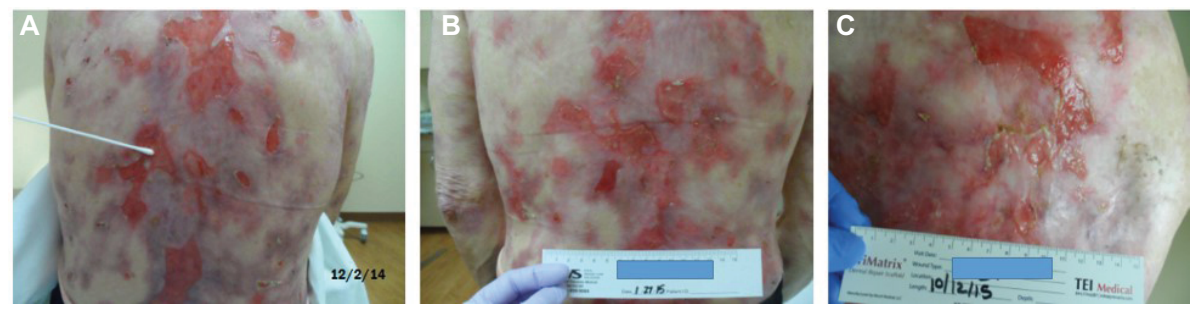

Figure 2 Wound response: central mid-back ulceration.

Note: (A-C) show improvement progress from baseline February 12, 2014 to February 10, 2015.
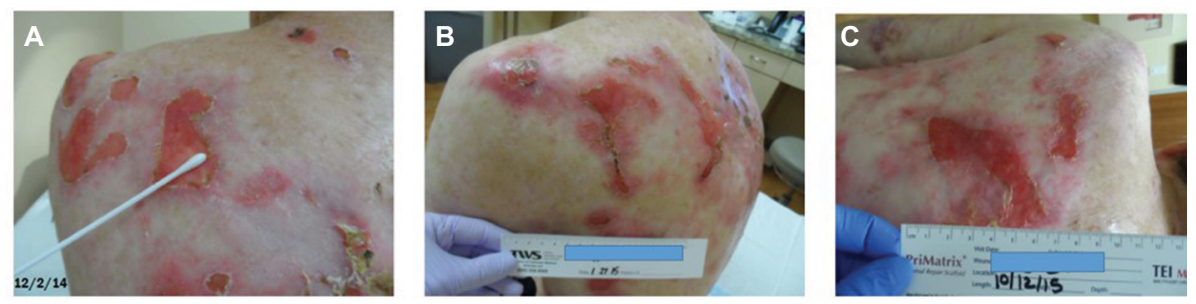

Figure 3 Wound response: distal mid-back ulceration.

Note: (A-C) show improvement progress from baseline February 12, 2014 to February 10, 2015. 

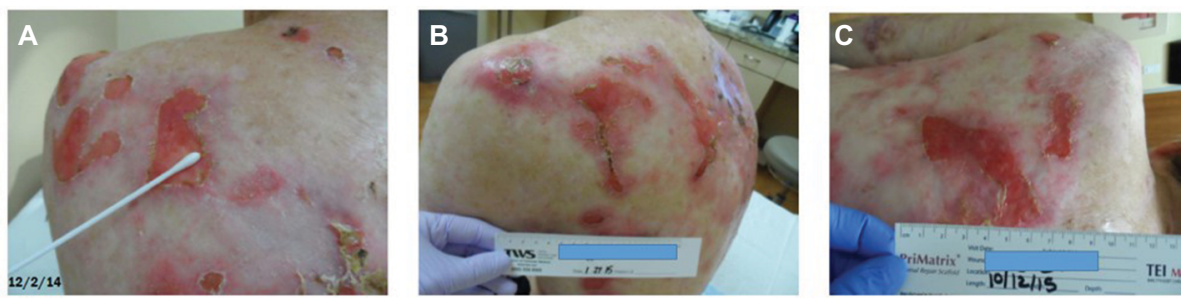

Figure 4 Wound response: left shoulder blade ulceration.

Note: (A-C) show improvement progress from baseline February 12, 2014 to February 10, 2015.

understanding of the genetic mutations and characteristic ultrastructural morphology that leads to blistering of the skin. However, there are few effective therapies for EB, and the treatment is primarily supportive and preventive.

This case is unique in that it describes the successful use of a multivalent wound-healing ointment in a patient with a long history of junctional EB. The ulcers under investigation had been persistent for over 60 years. He has some wounds that have been chronically non-healing for months due to their difficult location. The age of the wound may also be a factor. It is possible that the extensive wounds on his back are colonized differently. Due to the number of wounds on his body, he changed his dressings daily. While, the patient used a clean Q-tip to apply the ointment, contamination is possible, although of less concern with normal skin flora.

The patient showed a positive response within a few months of treatment with the wound care ointment and nutritional supplement. The healing response was most likely facilitated by the reduction in bacterial bioburden along with the elimination of fungal species. The ointment provided a moist wound-healing environment and reduced blistering and itching. Additionally, nutritional supplements that provide arginine and selected micronutrients, such as vitamin $\mathrm{C}$ and E, can help address deficiencies in a patient's diet to accelerate wound healing. ${ }^{12-14}$ This case has formed a strong basis for further research on the use of this wound care ointment as an adjunctive therapy in the management of EB.

The multivalent wound care ointment provides a novel option for an effective control of bioburden and moisture management in patients with EB. The combination of jojoba seed oil and organic beeswax provides lasting moisturization without the risk of periwound maceration. Inactive vehicle ingredients, jojoba seed oil and natural beeswax, can manage moisture without causing maceration in the wound or periwound area. ${ }^{15}$ Studies also have shown that jojoba oil facilitates cutaneous penetration of active ingredients. ${ }^{16,17}$

\section{Ethics statement}

A written authorization for release of information was obtained from the patient, and he consented to have the case details and any accompanying images published.

\section{Disclosure}

The authors report no conflicts of interest in this work.

\section{References}

1. Fine JD. Epidemiology of inherited epidermolysis bullosa. In: Fine JD, Hintner H, editors. Life with Epidermolysis Bullosa (EB): Etiology, Diagnosis, Multidisciplinary Care and Therapy. Wien, Austria: Springer-Verlag; 2009;25-29.

2. Hintner H, Stingl G, Schuler G, et al. Immunofluorescence mapping of antigenic determinants with the dermal-epidermal junction in the mechanobullous disease. J Invest Dermatol. 1981;76(2):113-118.

3. Fassihj H, McGrath JA. Prenatal diagnosis of epidermolysis bullosa. Dermatol Clin. 2010;28(2):231-237.

4. Hammersen J, Has C, Naumann-Bartsch N, et al. Genotype, clinical course, and therapeutic decison making in 76 infants with severe generalized junctional epidermolysis bullosa. $J$ Invest Dermatol. 2016;136(11):2150-2157.

5. Cooper TW, Bauer EA, Briggaman RA. The mechanobullous diseases (epidermolysis bullosa). In: Fitzpatrick TB, editor. Fitzpatrick's Dermatology in General Medicine. 3rd ed. New York: McGraw-Hill. 1987;610-626.

6. Coulombe PA, Fuchs E. Epidermolysis bullosa simplex. Semin Dermatol. 1993;12(3):173-190.

7. Rezniczek GA, de Pereda JM, Reipert S, Wiche G. Linking integrin $\alpha 6 \beta 4$-based cell adhesion to the intermediate filament cytoskeleton: direct interaction between the $\beta 4$ subunit and plectin at multiple molecular sites. J Cell Biol. 1998;141(1):209-225.

8. Walko G, Castañón MJ, Wiche G. Molecular architecture and function of the hemidesmosome. Cell Tissue Res. 2015;360(2):363-378.

9. Varki R, Sadowski S, Uitto J, Pfendner E. Epidermolysis bullosa. II. Type VII collagen mutations and phenotype-genotype correlations in the dystrophic subtypes. J Med Genet. 2007;44(3):181-192.

10. Siañez-González C, Pezoa-Jares R, Salas-Alanis JC. Epidermólisis Ampollosa Congénita : Revisión del Tema. [Congenital epidermolysis bullosa: a review]. Actas Dermosifiliogr. 2009;100(10):842-856. Spanish.

11. Aumilley M, Has C, Tunggal L, Bruckner-Tuderman L. Molecular basis of inherited skin-blistering disorders, and therapeutic implications. Expert Rev Mol Med. 2006;8(24):1-21.

12. Ellinger S. Micronutrients, arginine and glutamine: does supplementation provide an efficient tool for prevention and treatment of different kinds of wounds? Adv Wound Care. 2014;3(11):691-707.

13. Laura B. Evidence corner: nutrition benefits patients following trauma. Wounds. 2014;26(8):245-247. 
14. Soriano FL, Vazquez LMA, Maristany CP, Graupera JMX, WoutersWesseling W, Wagenaar L. The effectiveness of oral nutritional supplementation in the healing of pressure ulcers. $J$ Wound Care. 2004;13(8):319-322.

15. Pazyar N, Yaghoobi R, Ghassemi MR, Kazerouni A, Rafeie E, Jamshydian N. Jojoba in dermatology: a succinct review. G Ital Dermatol Venereol. 2013;148(6):687-691.
16. Wang LH, Wang CC, Kuo SC. Vehicle enhancer effects on human skin penetration of aminophylline from cream formulations: evaluation in vivo. J Cosmet Sci. 2007;58(3):245-254.

17. Serena T, Connell H, McConnell S, et al. Novel multivalent wound healing ointment provides bioburden control and moisture management: a retrospective registry data analysis. Adv Skin Wound Care. 2016;29(10): $461-468$.
Chronic Wound Care Management and Research is an international, peer reviewed, open access, online journal publishing original research, reviews, editorials, and commentaries on the causes and management of chronic wounds and the major issues related to chronic wound management. Topics also include chronic wounds as comorbidities to other conditions, patient adherence to therapy, and the economic burden of chronic wounds. The manuscript management system is completely online and includes a very quick and fair peer review system, which is all easy to use. Visit http://www.dovepress.com/testimonials.php to read real quotes from published authors.

Submit your manuscript here: https://www.dovepress.com/chronic-wound-care-management-and-research-journal 